\title{
SELF-MAPS OF LOOP SPACES. II
}

BY

\author{
H. E. A. CAMPBELl, F. R. COHEN, F. P. PETERSON AND P. S. SELICK ${ }^{1}$
}

\begin{abstract}
We study under what conditions on a finite CW complex $X$ is $Q(X)$ atomic.
\end{abstract}

1. Introduction. Let $Q(X)=\Omega^{\infty} \Sigma^{\infty}(X)$. In [3] we showed that $Q\left(S^{r}\right)$ is atomic at $p$ if $r \geqslant 2$ and that the universal covering space of $Q\left(S^{1}\right)$ is atomic at $p$. In this paper we study the question of what conditions one must have on a finite complex $X$ so that $Q(X)$ is atomic at 2. For example, we show in Theorem 2.5 that $Q\left(\Sigma C P^{n}\right)$ is atomic at 2 for all $n$. We conjecture that $Q\left(\Sigma^{s} X\right)$ is atomic at 2 if $s \geqslant 1$ and $X$ is "stably atomic". We can prove this only when $s \geqslant 2$ and $X$ is restricted to a special kind of CW-complex.

The proofs of our results depend heavily on the methods of [3], especially those used to prove the stable case. We use algebraic properties of the algebra of Dickson invariants in a crucial way in order to use an induction on the skeleta of $X$. This is done in \$3. We also note that something different happens when $X$ is no longer finite dimensional by showing that $Q\left(\Sigma C P^{\infty}\right)$ is not atomic at 2 (Theorem 2.7). Finally, we note the finite cases proved in [3] do not generalize by showing that $\Omega^{n} \Sigma^{n} X$ is not atomic at $p$ unless $X$ is a sphere or a point (Theorem 2.8), although [2] gives a different finite analogue.

A detailed statement of our results is given in $\$ 2$ and the proofs are given in $\S \S 3-7$.

2. Statement of results. We first define the term atomic and various related notions (see also [2, 3]).

Definition. An $(r-1)$-connected space $Y$ is called atomic if given any self-map $f: Y \rightarrow Y$ such that $f_{*}: H_{r}(Y) \rightarrow H_{r}(Y)$ is an isomorphism, then $f_{*}: H_{*}(Y) \rightarrow$ $H_{*}(Y)$ is an isomorphism. $Y$ is called $H$-atomic if $Y$ is an $H$-space and we are given only self- $H$-maps $f . Y$ is called atomic at $p$, where $p$ is a prime, if $Y$ has the same property with all homology groups having $Z / p Z$ coefficients. $Y$ is called $H$-atomic at $p$ if further, $Y$ is an $H$-space and we are given only self- $H$-maps $f$.

Definition. A connected space $X$ is called stably atomic (stably atomic at $p$ ) if $\Sigma^{s}(X)$ is atomic (atomic at $p$ ) for all $s \geqslant 0$.

\footnotetext{
Received by the editors February 19, 1985.

1980 Mathematics Subject Classification. Primary 55P47, 55P35, 55P45.

${ }^{1}$ The authors were partially supported by the N.S.F. and the Natural Science and Engineering Research Council of Canada.
} 
We first note the following simple proposition.

Proposition 2.1. If $Y$ is 1-connected and $\Omega Y$ is $H$-atomic ( $H$-atomic at $p$ ), then $Y$ is atomic (atomic at $p$ ).

We will be interested in finding conditions on $X$ that imply that $Q\left(\Sigma^{s} X\right)$ is atomic. Many of our results will be stated in the form: $Q(X)$ is $H$-atomic at $p$ under certain conditions on $X$. Since $\Omega Q(\Sigma X)$ is homotopy equivalent to $Q(X)$, Proposition 2.1 then implies that $Q(\Sigma X)$ is atomic at $p$.

The following proposition gives a necessary condition on $X$. We prove it in $\S 5$.

Proposition 2.2. If $Q\left(\Sigma^{s} X\right)$ is atomic at $p$ for some $s$, then $X$ is stably atomic at $p$.

We now assume that $p=2$; we presume there are similar theorems for $p$ odd. We also assume all homology and cohomology groups have coefficients $Z / 2 Z$. Before stating our main results, we recall $H_{*}(Q(X))$. Let $Q_{i}$ be the Dyer-Lashof (KudoAraki) operations defined for infinite loop spaces. Let $I=\left(i_{1}, \ldots, i_{k}\right)$ be a sequence of integers with $i_{j} \leqslant i_{j+1}$ and $i_{1}>0$. Let $Q_{I}=Q_{i_{1}} \cdots Q_{i_{k}}$, where $Q_{I}=$ id if $k=0$. Let $\left\{x_{i}\right\}$ be a basis for $H_{*}(X)$. Then $H_{*}(Q(X))=Z / 2 Z\left[Q_{I}\left(x_{i}\right)\right]$. Let $i_{*}: H_{*}(X)$ $\rightarrow H_{*}(Q(X))$ and $\pi: H_{*}(Q(X)) \rightarrow H_{*}(X)$ be the natural maps. Let $f: Q(X) \rightarrow$ $Q(X)$ be a self-map which induces an isomorphism on the lowest nonvanishing homology group. Let $x_{i}^{\prime}=\pi f_{*} i_{*}\left(x_{i}\right)$. If $X$ is stably atomic at 2 , then $\left\{x_{i}^{\prime}\right\}$ is also a basis for $H_{*}(X)$.

We need to put a condition on our CW-complexes $X$. A CW-complex is called special (at 2) if it is 2-homotopy equivalent to a CW-complex $X$ with $C_{*}(X)=$ $H_{*}(X)$, that is, if there is a CW-structure with zero boundaries. (Using homology decompositions (see Moore [17]) and the fact that Moore spaces for finitely generated groups are special, it is not hard to see that a simply-connected CW-complex of finite type is special.)

In $§ 3$ we study the Dickson algebra and prove the following general theorem.

TheOrem 2.3. Let $X$ be a finite $C W$-complex, which is special. Assume that $X$ is stably atomic at 2. Let $f: Q(X) \rightarrow Q(X)$ be an H-map. Assume that $f_{*}\left(Q_{1} x_{i}\right)=$ $Q_{1} x_{i}^{\prime}+$ other terms and that $f_{*}\left(Q_{1} Q_{1} x_{i}\right)=Q_{1} Q_{1} x_{i}^{\prime}+$ other tems. (Here $x_{i}$ and $x_{i}^{\prime}$ are as above, and other terms mean terms of another filtration in the given basis.) Then $f_{*}$ is an isomorphism.

Since double loop maps commute with $Q_{1}$, we get the following corollary.

Corollary 2.4. Let $X$ be a finite $C W$-complex, which is special. Assume $X$ is stably atomic at 2. Then $Q\left(\Sigma^{s} X\right)$ is atomic at 2 if $s \geqslant 2$.

In $\S 4$, we apply Theorem 2.3 to projective spaces and prove the following theorem.

THEOREM 2.5. $Q\left(C P^{n}\right)$ is $H$-atomic at 2 for all $n . Q\left(R P^{n}\right)$ is $H$-atomic at 2 for $n \neq 3$ or 7 .

In $\S 5$, we apply Theorem 2.3 to prove a general theorem for finite $\mathrm{CW}$-complexes $X$. 
THEOREM 2.6. Let $X$ be a finite $C W$-complex which is special and which is stably atomic at 2. Assume that all elements of $H_{*}(X)$ are primitive. Furthermore, assume $H^{*}(X)$ is a trivial A-module. Then $Q(X)$ is $H$-atomic at 2.

On the basis of our results, we offer a conjecture.

Conjecture. Let $X$ be a connected finite CW-complex. Assume $X$ is stably atomic at 2. Then $Q\left(\Sigma^{s} X\right)$ is atomic at 2 if $s \geqslant 1$.

This conjecture is not true when $X$ is an infinite dimensional CW-complex. In $\S 6$ we use the results of [3] to study $R P^{\infty}$ and $C P^{\infty}$.

THEOREM 2.7. $Q\left(\Sigma R P^{\infty}\right)$ and $Q\left(\Sigma C P^{\infty}\right)$ are not atomic at 2 .

The proof of this theorem is a corollary of our discussion of the Kahn-Priddy theorem [15] in $\S 6$.

We have studied some finite versions of $Q(X)$ to see if they could be atomic at 2 . [2] gives our results for $\operatorname{Map}_{*}\left(P^{3}(2), S^{n}\right)$, and in $\S 7$, we prove the following theorem.

THEOREM 2.8. If $\Omega^{n} \Sigma^{n} X$ is atomic at $p$ for $n \geqslant 2$, then $H_{*}(X ; Z / p Z)=$ $H_{*}(p t ; Z / p Z)$ or $H_{*}\left(S^{q} ; Z / p Z\right)$ for some $q$.

3. The Dickson algebra and the proof of 2.3. Let $f: Q(X) \rightarrow Q(X)$ be a self- $H$-map. To show that $f_{*}: H_{*}(Q X) \rightarrow H_{*}(Q X)$ is an isomorphism it is enough to show that the induced map on $Q H_{*}(Q X)$, the module of indecomposables, is an isomorphism. (This is because $f_{*}$ is multiplicative.)

Let $Q_{k}(X) \subset Q H_{*}(Q X)$ be the subspace generated by Dyer-Lashof operations of length $k$ on $H_{*}(X)$ modulo those starting with $Q_{0} . R[k]$ denotes the Dyer-Lashof operations of length $k$. Recall $[3, \S 1]$ that $R[k]^{*}=Z / 2 Z\left[a_{1}, \ldots, a_{k}\right]$, the algebra of Dickson invariants. The following two propositions relate $Q_{k}\left(S^{r}\right)$ to the Dickson algebra. Here $R[k] /\left(Q_{0}, \ldots, Q_{r}\right)$ denotes the Dyer-Lashof operations of length $k$ all of whose subscripts are $>r$.

Proposition 3.1. $Q_{k}\left(S^{r}\right) \rightarrow \Sigma^{r}\left(R[k] /\left(Q_{0}, \ldots, Q_{r}\right)\right)$ given by $Q_{i_{1}} \cdots Q_{i_{k}}\left(x_{r}\right) \mapsto$ $Q_{i_{1}+r} \cdots Q_{i_{k}+r}$ is an isomorphism of $A$-modules.

Proposition 3.2. $\left(R[k] /\left(Q_{0}, \ldots, Q_{r}\right)\right)^{*}=\left(a_{k}^{r+1}\right) \subset Z / 2 Z\left[a_{1}, \ldots, a_{k}\right]$.

3.1 is an easy application of the Nishida formula and 3.2 is proved in the same way as Theorem 1.7 of [3].

Definition. An $\mathscr{A}$-module is $\mathscr{A}$-atomic if given an $\mathscr{A}$-map $f: M \rightarrow M$ which is an isomorphism in the lowest nontrivial dimension, then $f$ is an isomorphism.

A key fact is the following proposition, which is proved below.

Proposition 3.3. Let $M=\Pi_{k}\left\langle a_{k}^{r+1}\right\rangle$ and let $f: M \rightarrow M$ be an $\mathscr{A}$-map such that $f\left(a_{k}^{r+1}\right)=a_{k}^{r+1}+$ others for all $k$. Then $f$ is an isomorphism. In particular, $\left\langle a_{k}^{r+1}\right\rangle$ is $\mathscr{A}$-atomic.

We now start the proof of Theorem 2.3. Let $X^{r}$ be the $r$-skeleton of $X$. Let $P(X)$ denote $P H^{*}(Q X)=Q H_{*}(Q X)^{*}=\Pi_{k} Q_{k}(X)^{*}$. We want to show that $f^{*}: P(X)$ $\rightarrow P(X)$ is an isomorphism. We first want to prove by downward induction that $f^{*}$ induces a map $f_{r}: P\left(X^{r}\right) \rightarrow P\left(X^{r}\right)$. We will then prove by upward induction that $f_{r}$ is an isomorphism. Let $Q_{1}^{k}=Q_{1} \cdots Q_{1} \in R[k]$. 
LEMMA 3.4. Under the hypotheses of $2.3, f_{*}\left(Q_{1}^{k} x_{i}\right)=Q_{1}^{k} x_{i}^{\prime}+$ others.

Proof. The hypotheses of 2.3 include this condition for $k \leqslant 2$. Assume $k>2$, and prove this by induction on $k$. We note that "others" must be primitive. We have $\mathrm{Sq}_{*}^{1} Q_{1}^{k} x_{i}=Q_{0} Q_{1}^{k-1} x_{i}$. Since $k>2$, no other basis element has this property. Hence, $f_{*}\left(Q_{1}^{k} x_{i}\right)=Q_{1}^{k} x_{i}^{\prime}+$ primitives.

Lemma 3.5. Let $x \in P\left(X^{r} / X^{r-1}\right),|x|=t$. Then $x \in \bigcap_{i>t-r+2} \operatorname{Ker~Sq}$.

Proof. $P\left(X^{r} / X^{r-1}\right)=\oplus P\left(S^{r}\right)=\oplus \prod_{k} \Sigma^{r}\left(a_{k}^{r+1}\right)$ by Propositions 3.1 and 3.2. Thus $x=\sigma^{r} y$ with $|y|=t-r$. Since $\left(a_{k}^{r+1}\right)$ is an unstable $\mathscr{A}$-module, $y \in$ $\bigcap_{i>t-r+2} \mathrm{Ker} \mathrm{Sq}^{i}$.

Lemma 3.6. $0 \rightarrow P\left(X^{r} / X^{r-1}\right) \rightarrow P\left(X^{r}\right) \rightarrow P\left(X^{r-1}\right) \rightarrow 0$ is exact.

Proof. The cofibration $X^{r-1} \rightarrow X^{r} \rightarrow X^{r} / X^{r-1}$ induces a fibration $Q\left(X^{r-1}\right) \rightarrow$ $Q\left(X^{r}\right) \rightarrow Q\left(X^{r} / X^{r-1}\right)$ and it is easy to see that the Serre spectral sequence of this fibration collapses. The lemma now follows.

Lemma 3.7. Let $0 \neq x \in P\left(X^{r-1}\right),|x|=t$. Then $\mathrm{Sq}^{i}(x) \neq 0$ for some $i>t-r+$ 2.

Proof. By Lemma 3.6, either $x \in P\left(X^{r-1} / X^{r-2}\right)$ or $0 \neq x \in P\left(X^{r-2}\right)$. Continue in this manner and find $s<r$ such that $x$ projects to a nonzero element in $P\left(X^{s} / X^{s-1}\right)$. Then $0 \neq \mathrm{Sq}^{t-s}(x) \in P\left(X^{s} / X^{s-1}\right)$ and hence in $P\left(X^{r-1}\right)$. This is because $P\left(X^{s} / X^{s-1}\right)=\oplus \Pi_{k} \Sigma^{s}\left(a_{k}^{s+1}\right)$ by Propositions 3.1 and 3.2 , and $\mathrm{Sq}^{t-s}$ is a monomorphism in degree $t-s$ of the Dickson algebra [3]. Since $s<r$, we have $t-s>t-r$.

We return to the proof of 2.3. We assume that we have $f_{r}: P\left(X^{r}\right) \rightarrow P\left(X^{r}\right)$ which is a map of $\mathscr{A}$-modules. This certainly exists when $r=\operatorname{dim} X$. We want to fill in the following diagram by maps of $\mathscr{A}$-modules:

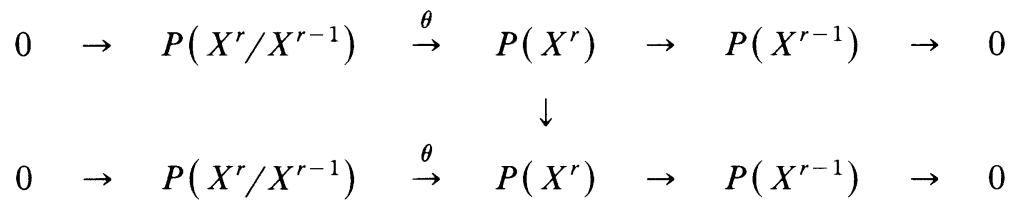

By Lemmas 3.5 and 3.7, $\operatorname{Im} \theta=\bigcap_{i>t-r} \operatorname{Ker~Sq}^{i}$ and hence it is preserved by $f_{r}$. This defines $f_{r-1}$ on $P\left(X^{r-1}\right)$ and makes a commutative ladder. By induction we know that $f_{r-1}$ is an isomorphism and thus so is $f_{r}$ if $f_{r} \mid P\left(X^{r} / X^{r-1}\right)$ is an isomorphism. (We write $f_{r} \mid$ for $f_{r} \mid P\left(X^{r} / X^{r-1}\right)$.) The bottom classes of $P\left(X^{r} / X^{r-1}\right)$ are dual to $Q_{1}^{k} x_{i}$ and thus by Lemma 3.4, $f_{r}$ preserves the classes $a_{k}^{r+1}$ (at this point we use the fact that $X$ is special). By Proposition 3.3, $f_{r} \mid$ is an isomorphism and the five lemma shows that $f_{\operatorname{dim} X}$ is an isomorphism, and the proof of 2.3 is complete.

We now prove Proposition 3.3. The idea of the proof is similar to that of Corollary 4.2 of [3]. Let $x \in \Pi_{k}\left\langle a_{k}^{r+1}\right\rangle$ be in $\operatorname{Ker} f$. Using the techniques of Corollary 4.2 of [3], we can find a Steenrod operation $\alpha$ such that $\alpha(x)=a_{k}^{2^{s}}$ for some $s$ and some $k$, and $\alpha(x) \in \operatorname{Ker} f$. However, there is a Steenrod operation $\psi$ such that $\psi\left(a_{k}^{r+1}\right)=a_{k}^{2^{s}}$ and so $a_{k}^{2^{s}}$ is not in $\operatorname{Ker} f$. 
4. $Q\left(C P^{n}\right)$ and $Q\left(R P^{n}\right)$. We first study $Q\left(C P^{n}\right)$. Let $x_{2 i} \in H_{2 i}\left(C P^{n}\right)$ be the generators. To apply Theorem 2.3 we must show that $f_{*}\left(x_{2 i}\right)=x_{2 i}+$ of, $f_{*}\left(Q_{1} x_{2 i}\right)$ $=Q_{1} x_{2 i}+$ of, and $f_{*}\left(Q_{1} Q_{1} x_{2 i}\right)=Q_{1} Q_{1} x_{2 i}+$ of for $i \leqslant n$. ("of" stands for other filtrations.) These facts will be proved in Lemmas 4.1-4.5.

LEMMA 4.1. If $f_{*}\left(x_{2 i}\right)=x_{2 i}+$ of, then $f_{*}\left(Q_{2} x_{2 n}\right)=Q_{2} x_{2 n}+$ of. Further, if $n$ is odd, then $f_{*}\left(Q_{2} x_{2 n-2}\right)=Q_{2} x_{2 n-2}+$ of.

Proof. Case $1, n$ even. $\left|Q_{2} x_{2 n}\right|=4 n+2$, so there are no decomposable elements of filtration 2 in that dimension. Hence $f_{*}\left(Q_{2} x_{2 n}\right)=\sum a_{2 i} Q_{4 n+2-4 i} x_{2 i}+$ of. Apply $\mathrm{Sq}_{*}^{2}$ to get $0=\sum i a_{2 i} Q_{4 n-4 i} x_{2 i}+$ of. Hence, $a_{2 i}=0$ if $i$ is odd. We now apply $\mathrm{Sq}_{*}^{4}$ to the same equation to get

$$
f_{*}\left(Q_{2} x_{2 n-2}\right)=a_{2 n} Q_{2} x_{2 n-2}+\sum a_{4 j}\left(j Q_{4 n-2-8 j} x_{4 j}+Q_{4 n+2-8 j} x_{4 j-2}\right)+\text { of. }
$$

Apply $\mathrm{Sq}_{*}^{2}$ to this last equation to get

$$
f_{*}\left(Q_{0} x_{2 n-2}\right)=a_{2 n} Q_{0} x_{2 n-2}+\sum a_{4 j} Q_{4 n-8 j} x_{4 j-2}+\text { of. }
$$

Since $f_{*}\left(x_{2 n-2}\right)=x_{2 n-2}+$ of and $f_{*}$ is multiplicative, we see that $a_{2 n}=1$ and $a_{4 j}=0, j<(n / 2)-1$.

Case 2, $n$ odd. As before, apply $\mathrm{Sq}_{*}^{2}$ to $f_{*}\left(Q_{2} x_{2 n}\right)$ to get $f_{*}\left(Q_{0} x_{2 n}\right)=$ $\sum i a_{2 i} Q_{4 n-4 i} x_{2 i}+$ of. Hence $a_{2 n}=1$ and $a_{2 i}=0$ if $i$ is odd, $i<n$. As before, apply $\mathrm{Sq}_{*}^{2} \mathrm{Sq}_{*}^{4}$ to $f_{*}\left(Q_{2} x_{2 n}\right)$ to get $0=\sum a_{4 j} Q_{4 n-8 j} x_{4 j-2}+$ of. Hence $a_{4 j}=0, j \leqslant$ $(n-1) / 2$, and we have shown Case 2 .

Finally, consider

$$
f_{*}\left(Q_{2} x_{2 n-2}\right)=\sum a_{2 i} Q_{4 n-4 i-2} x_{2 i}+\text { of }+b x_{2 n-2} \cdot x_{2 n} .
$$

Apply $\mathrm{Sq}_{*}^{2}$ to get $0=\sum i a_{2 i} Q_{4 n-4 i-4} x_{2 i}+b x_{2 n-4} \cdot x_{2 n}+$ of. Hence $b=0$ and $a_{2 i}=0$ if $i$ is odd. Now continue as in Case 1 .

LEMMA 4.2. If $f_{*}\left(x_{2 i}\right)=x_{2 i}+$ of, all $i$, then $f_{*}\left(Q_{1} x_{2 i}\right)=Q_{1} x_{2 i}+$ of, all $i$.

Proof. By Lemma 4.1, $f_{*}\left(Q_{2} x_{2 n}\right)=Q_{2} x_{2 n}+$ of. Apply $\mathrm{Sq}_{*}^{1}$ to see that $f_{*}\left(Q_{1} x_{2 n}\right)=Q_{1} x_{2 n}+$ of. We now use downward induction on $i$. Assume the lemma is true for $i>k$.

Case $1, k$ odd. Then $\mathrm{Sq}_{*}^{4} Q_{1} x_{2(k+1)}=Q_{1} x_{2 k}$ and this case follows easily by induction.

Case 2, $k$ even. $f_{*}\left(Q_{2} x_{2 k}\right)=\sum b_{2 j} Q_{4 k+2-4 j} x_{2 j}+$ decomp. + of. Apply $\mathrm{Sq}_{*}^{2}$ to deduce that $b_{2 j}=0$ if $j$ is odd. Now apply $\mathrm{Sq}_{*}^{4} \mathrm{Sq}_{*}^{1}$ to get

$f_{*}\left(Q_{1} x_{2 k-2}\right)=\sum b_{4 j}\left[(1+j) Q_{4 k-3-8 j} x_{4 j}+Q_{4 k+1-8 j} x_{4 j-2}\right]+b_{2 k} Q_{1} x_{2 k-2}+$ of.

Case $2 \mathrm{a}, k \equiv 0 \bmod 4$. Since $\mathrm{Sq}_{*}^{4} x_{2 k+2}=x_{2 k-2}$, by induction we have

$$
f_{*}\left(\mathrm{Sq}_{*}^{8} Q_{1} x_{2 k+2}\right)=\mathrm{Sq}_{*}^{8}\left(Q_{1} x_{2 k+2}+\text { of }\right)=Q_{1} x_{2 k-2}+\text { of }=f_{*}\left(Q_{1} x_{2 k-2}\right) \text {. }
$$

Thus $b_{2 k}=1$ and $b_{4 j}=0$ if $j<k / 2$. Note that $x_{2 k+2}$ exists because $k<n$.

Case $2 \mathrm{~b}, k \equiv 2 \bmod 4$. Then $\mathrm{Sq}_{*}^{4} x_{2 k+4}=x_{2 k}$. Hence the induction hypothesis gives $f_{*}\left(Q_{1} x_{2 k}\right)=f_{*}\left(\mathrm{Sq}_{*}^{8} Q_{1} x_{2 k+4}\right)=Q_{1} x_{2 k}+$ of. Note that $x_{2 k+4}$ exists as $k \leqslant n$ -2 if $n$ is even and $k \leqslant n-3$ if $n$ is odd by Lemma 4.1. 
LEMMA 4.3. If $f_{*}\left(x_{2 i}\right)=x_{2 i}+$ of, all $i$, then $f_{*}\left(Q_{1} Q_{1} x_{2 i}\right)=Q_{1} Q_{1} x_{2 i}+$ decomp. + of.

Proof.

$$
\begin{aligned}
f_{*}\left(Q_{2} Q_{2} x_{2 i}\right)= & \sum a_{k j r} Q_{2 k} Q_{2 j} x_{2 r}+\sum b_{k r j s} Q_{2 k} x_{2 r} \cdot Q_{2 j} x_{2 s} \\
& +\sum c_{k r j s} Q_{2 k+1} x_{2 r} \cdot Q_{2 j-1} x_{2 s}+\sum d_{k j r} Q_{2 k} Q_{2 j+1} x_{2 s} \\
& + \text { squares }+ \text { (products of length } \geqslant 3)+ \text { of. }
\end{aligned}
$$

Apply $\mathrm{Sq}_{*}^{1} \mathrm{Sq}_{*}^{2} \mathrm{Sq}_{*}^{1}$ to get

$$
\begin{aligned}
& f_{*}\left(Q_{0} Q_{1} x_{2 k}\right)=\sum a_{k j r} Q_{2 k-2} Q_{2 j-1} x_{2 r} \\
& +\sum b_{k r j s}\left[(k+r) Q_{2 k-3} x_{2 r} \cdot Q_{2 j-1} x_{2 s}+(j+s+1) Q_{2 k-1} x_{2 r}\right. \\
& \cdot Q_{2 j-3} x_{2 s}+(k+s+1) Q_{2 k-3} x_{2 r} \cdot Q_{2 j-1} x_{2 j} \\
& \left.\quad+(j+s) Q_{2 k-1} x_{2 r} \cdot Q_{2 j-3} x_{2 s}\right]+(\text { length } \geqslant 3)+\text { of. }
\end{aligned}
$$

By Lemma 4.2, $f_{*}\left(Q_{0} Q_{1} x_{2 i}\right)=Q_{0} Q_{1} x_{2 i}+$ of. Comparing coefficients gives $a_{11 i}=1$ and $a_{k j r}=0$ otherwise.

LEMMA 4.4. Let $n \neq 2^{t}-1$. If $f_{*}\left(x_{2}\right)=x_{2}$, then $f_{*}\left(x_{2 i}\right)=x_{2 i}+$ of, all $i$.

Proof. $H_{*}\left(C P^{n}\right) \rightarrow H_{*}\left(Q C P^{n}\right) \rightarrow H_{*}\left(Q C P^{n}\right) \rightarrow H_{*}\left(C P^{n}\right)$ is a map of $A$-modules. Since $H_{*}\left(C P^{n}\right)$ is $A$-atomic, the lemma follows.

LEMMA 4.5. Let $n=2^{t}-1$. If $f_{*}\left(x_{2}\right)=x_{2}$, then $f_{*}\left(x_{2 i}\right)=x_{2 i}+$ of, all $i$.

Proof. $H_{*}\left(C P^{n-1}\right) \rightarrow H_{*}\left(C P^{n}\right) \rightarrow H_{*}^{r}\left(Q C P^{n}\right) \rightarrow H_{*}\left(Q C P^{n}\right) \rightarrow H_{*}\left(C P^{n-1}\right)$ is a map of $A$-modules. Hence, by Lemma 4.4, this lemma is true for $i<n . \psi\left(x_{2 i}\right)=$ $\sum x_{2 i} \otimes x_{2 n-2 i}$. We have that

$$
\psi f_{*}\left(x_{2 n}\right)=\sum f_{*}\left(x_{2 i}\right) \otimes f_{*}\left(x_{2 n-2 i}\right)=\sum x_{2 i} \otimes x_{2 n-2 i}+\text { others. }
$$

The only elements having this diagonal are $x_{2 n}+$ of or $\sum x_{2 i} \cdot x_{2 n-2 i}+$ others. If the latter, then apply $\mathrm{Sq}_{*}^{2}$ to get $0=Q_{0} x_{2^{t}-2}+$ others. Since only $Q_{2} x_{2^{t}-2}$ goes to $Q_{0} x_{2^{t}-2}$ by $\mathrm{Sq}_{*}^{2}$ besides $x_{2^{t}-2} \cdot x_{2^{t}}$, we have $f_{*}\left(x_{2 n}\right)=\sum x_{2 i} \cdot x_{2 n-2 i}+Q_{2^{2}} x_{2^{t}-2}+$ others. Apply $\mathrm{Sq}_{*}^{1}$ to get $0=0+Q_{1} x_{2^{t}-2}+$ others and others cannot cancel $Q_{1} x_{2^{t}-2}$. Hence the lemma is proved.

We now study $Q\left(R P^{n}\right)$. The proof is very analogous to that for $Q\left(C P^{n}\right)$ and we state the appropriate lemmas and give a short outline of the proof of each.

LEMma 4.6. If $f_{*}\left(x_{i}\right)=x_{i}+$ of, then $f_{*}\left(Q_{1} x_{n}\right)=Q_{1} x_{n}+$ of. Further, if $n$ is odd, then $f_{*}\left(Q_{1} x_{n-1}\right)=Q_{1} x_{n-1}+$ of.

Proof. Case 1, $n$ even. $f_{*}\left(Q_{1} x_{n}\right)=\sum a_{i} Q_{2 n-2 i+1} x_{i}+$ of. Apply $\mathrm{Sq}_{*}^{1}$ to deduce $a_{i}=0$ if $i$ is odd. Then apply $\mathrm{Sq}_{*}^{1} \mathrm{Sq}_{*}^{2}$ to deduce that $a_{n}=1$ and $a_{2 j}=0$ if $2 j<n$.

Case 2, $n$ odd. Apply $\mathrm{Sq}_{*}^{1}$ to deduce that $a_{i}=0$ for $i$ odd, $i<n$, and $a_{n}=1$. Apply $\mathrm{Sq}_{*}^{2}$ to deduce that $a_{2 j}=0,2 j \leqslant n-1$. Finally, consider $f_{*}\left(Q_{1} x_{n-1}\right)$. Apply $\mathrm{Sq}_{*}^{1}$ to deduce that $b=0$ and $a_{i}=0$ for $i$ odd, and then continue as in Case 1. 
LEMMA 4.7. If $f_{*}\left(x_{i}\right)=x_{i}+$ of, then $f_{*}\left(Q_{1} x_{i}\right)=Q_{1} x_{i}+$ of + decomp.

Proof. By Lemma 4.6, this is true for $i=n$. Assume true for $i>k$.

Case $1, k$ odd. Since $\mathrm{Sq}_{*}^{2} Q_{1} x_{k+1}=Q_{1} x_{k}$, this case follows immediately by downward induction.

Case 2, $k$ even. $f_{*}\left(Q_{1} x_{k}\right)=\sum b_{j} Q_{2 k-2 j+1} x_{j}+$ decomp. + of. Apply $\mathrm{Sq}_{*}^{1}$ to deduce that $b_{j}=0$ if $j$ is odd. Now apply $\mathrm{Sq}_{*}^{3}+\mathrm{Sq}_{*}^{1} \mathrm{Sq}_{*}^{2}$ to deduce $b_{2 j}=0$ but we cannot conclude that $b_{k}=1$ because $\left(\mathrm{Sq}_{*}^{3}+\mathrm{Sq}_{*}^{1} \mathrm{Sq}_{*}^{2}\right)\left(x_{k-1} \cdot x_{k+2}\right)=Q_{0} x_{k-1}$.

Case $2 \mathrm{a}, k \equiv 2 \bmod 4$. Then $\mathrm{Sq}_{*}^{4} Q_{1} x_{k+2}=Q_{1} x_{k}$ and we use "further" in Lemma 4.6 and induction.

Case $2 \mathrm{~b}, k \equiv 0 \bmod 4$. Apply $\mathrm{Sq}_{*}^{2}$ to $f_{*}\left(Q_{1} x_{k}\right)$ and $\mathrm{Sq}_{*}^{4}$ to $f_{*}\left(Q_{1} x_{k+1}\right)$ to show no decomp. appear and hence $b_{k}=1$.

LEMmA 4.8. If $f_{*}\left(Q_{1} x_{i}\right)=Q_{1} x_{i}+$ decomp. + of, then $f_{*}\left(Q_{1} Q_{1} x_{i}\right)=Q_{1} Q_{1} x_{i}+$ decomp. + of.

Proof. Case 1 , $i$ odd. $f_{*}\left(Q_{1} Q_{1} x_{i}\right)=a Q_{1} Q_{1} x_{i}+$ others. Apply $\mathrm{Sq}_{*}^{1}$ to deduce that $a=1$ because $\operatorname{Sq}_{*}^{1}\left(Q_{1} x_{i} \cdot Q_{2} x_{i}\right)=Q_{0} x_{i} \cdot Q_{2} x_{i}$.

Case 2, $i$ even. $f_{*}\left(Q_{1} Q_{2} x_{i}\right)=\sum a_{i-j} Q_{1} Q_{2+2 j} x_{i-j}+b Q_{3} Q_{3} x_{i-1}+\sum c Q_{I} x_{j}+$ decomp. + of. Apply $\mathrm{Sq}_{*}^{4} \mathrm{Sq}_{*}^{2}$ to deduce $a_{i}=1$ by Case 1 .

LEMMA 4.9. Let $n \neq 2^{t}-1$. If $f_{*}\left(x_{1}\right)=x_{1}$, then $f_{*}\left(x_{i}\right)=x_{i}+$ of, all $i$.

Proof. The proof is the same as for Lemma 4.4.

LEMMA 4.10. Let $n=2^{t}-1, t \geqslant 4$. If $f_{*}\left(x_{1}\right)=x_{1}$, then $f_{*}\left(x_{i}\right)=x_{i}+$ of, all $i$.

Proof. The proof of 4.5 does not generalize to the real case. For example, if $n=3$, then $x_{3}+x_{1} \cdot x_{2}+\left(x_{1}\right)^{3}+Q_{1} x_{1}$ could be in $\operatorname{Ker} f_{*}$. (Indeed, there is a homotopy equivalence $g: \Sigma^{2} R P^{3} \rightarrow \Sigma^{2}\left(R P^{2} \cup S^{3}\right)$ and thus $Q\left(R P^{3}\right)$ is homotopy equivalent to $Q\left(R P^{2} \cup S^{3}\right)$ by a map $\Omega^{2} Q(g)$.) We first prove that $f_{*}$ is an isomorphism in dimensions $<n$. The first step in the proof of Lemma 4.5 works, so we know that $f_{*}\left(x_{i}\right)=x_{i}+$ of for $i<n$. Then we note that the proof of Lemma 4.6 shows that if $f_{*}\left(x_{i}\right)=x_{i}+$ of for $i<n$, then $f_{*}\left(Q_{1} x_{n-1}\right)=Q_{1} x_{n-1}+$ of. Using this, we note that the induction in the proof of Lemma 4.7 still works in dimensions $<n$, as does the proof of 4.8. Next, we examine the proof of 2.3 in $\$ 3$. The induction which constructs $f_{r}$ is correct in our case and the only step that fails is the induction from $X^{n-1}$ to $X^{n}$, and it fails for $Q_{0}\left(X^{n}\right)$, i.e. it fails first in dimension $n$.

If $\operatorname{Ker} f_{*}=0$ in dimension $n$, Lemma 4.10 is proved. If $\operatorname{Ker} f_{*} \neq 0$ in dimension $n$, then an element of the form $y=x_{n}+\sum x_{i} \cdot x_{n-i}+$ others $\in \operatorname{Ker} f_{*}$. As in the proof of 3.2 in [2], we let $M$ be the mapping telescope of $f$ and let $f^{\prime}: Q\left(R P^{n}\right) \rightarrow M$. Let $F$ be the fibre of $f^{\prime} . F$ is $(n-1)$-connected and hence $y \in \operatorname{Ker} f_{*}$ is a spherical element. By taking an adjoint of this spherical class we see that the top cell of $R P^{n}$ is stably spherical and this can only happen in Hopf invariant 1 cases, i.e. when $t \leqslant 3$ (see [13]).

5. Proofs of Proposition 2.2 and Theorem 2.6. We first prove Proposition 2.2. It is enough to prove that $\Sigma^{N} X$ is atomic for large $N$. Let $g: \Sigma^{N} X \rightarrow \Sigma^{N} X$ be such that $g_{*}$ is an isomorphism on the bottom dimension, but $g_{*}: H_{*}\left(\Sigma^{N} X\right) \rightarrow H_{*}\left(\Sigma^{N} X\right)$ is 
not an isomorphism. Let $x \in H_{*}(X), x \neq 0, g_{*}\left(\sigma^{N} x\right)=0$. Choose $N>s$ and $N>|x|$. Then $g$ induces $f=Q(g): Q \Sigma^{N} X \rightarrow Q \Sigma^{N} X$ such that $f_{*}$ is an isomorphism on the bottom dimension, but $f_{*}$ is not an isomorphism because $H_{*}\left(Q \Sigma^{N} X\right)$ $=H_{*}\left(\Sigma^{N} X\right)$ if $* \leqslant\left|\sigma^{N} x\right|$. Hence $f$ is not a homotopy equivalence at $p$ and thus $\Omega^{N-s} f$ is also not a homotopy equivalence at $p$.

We now examine an attempt to prove the conjecture in $\$ 2$. If we assume that every element of $H_{*}(X)$ is primitive, i.e. that $H^{*}(X)$ has no nontrivial cup products, then the proof of Lemma 3.4 shows that we only need assume that $f_{*}\left(Q_{1} x_{i}\right)=Q_{1} x_{i}^{\prime}+$ others terms to apply the argument for Theorem 2.3. We now show that the assumptions of Theorem 2.6 are enough to prove this. Let $\left|x_{i}\right|=2^{s} \bmod 2^{s+1}$. Then $\mathrm{Sq}^{2^{s}} Q_{2^{s}} x_{i}=Q_{0} x_{i}$. Since $x_{i}^{\prime}$ is not in the image of any Steenrod operation, we get $f_{*}\left(Q_{2^{s}} x_{i}\right)=Q_{2^{s}} x_{i}^{\prime}+$ others. Apply $\mathrm{Sq}_{*}^{1} \mathrm{Sq}_{*}^{2} \cdots \mathrm{Sq}_{*}^{2^{s-1}}$ to this equation to get $f_{*}\left(Q_{1} x_{i}\right)=Q_{1} x_{i}^{\prime}+$ others. This proves Theorem 2.6.

A more delicate analysis would show that we can weaken the assumptions of Theorem 2.6, but the results are fragmentary.

6. Analogues of the Kahn-Priddy theorem. Let $X$ have the homotopy type of a connected CW-complex of finite type. If $X$ is not 1-connected, we assume that $X$ is an $H$-space. The following lemma is easily proved using the Whitehead theorem.

Lemma 6.1. If $f: X \rightarrow Y$ and $g: Y \rightarrow X$, with $(g f)_{*}$ an isomorphism in the bottom dimension and $X$ atomic at $p$, then $X$ is a retract of $Y$ at $p$.

Theorem 2.7 and the Kahn-Priddy theorem follow from the following corollary. $\left(Q^{\wedge}\left(S^{1}\right)\right.$ denotes the universal covering space of $Q\left(S^{1}\right)$.)

COROLlary 6.2. Let (1) $g_{1}: Q\left(\Sigma R P^{\infty}\right) \rightarrow Q^{\wedge}\left(S^{1}\right)$, (2) $g_{2}: Q\left(\Sigma C P^{\infty}\right) \rightarrow S U$, and (3) $g_{3}: \operatorname{Map}_{*}\left(P^{4}(2), S^{5}\right) \rightarrow \Omega_{0}^{3} S^{3}$ be maps which induce isomorphisms on the first nonvanishing homotopy group of the domain. Then there exist maps $f_{i}$ such that $g_{i} f_{i}$ is a homotopy equivalence at 2.

Proof. Since all three ranges of $g_{i}$ are atomic at 2, it suffices to exhibit maps $f_{i}$ and then apply Lemma 6.1. $\left(Q^{\wedge}\left(S^{1}\right)\right.$ is atomic at 2 by [3], $\Omega_{0}^{3} S^{3}$ is atomic at 2 by [2], and it is easily checked that $S U$ is atomic at 2.) To define $f_{1}$, consider the second stable Hopf invariant $h_{2}: Q\left(S^{1}\right) \rightarrow Q\left(D_{2}\left(S^{1}\right)\right)$ [14]. $D_{2}\left(S^{1}\right)$ is homotopy equivalent to $\Sigma R P^{\infty}$ and $Q\left(S^{1}\right)$ is homotopy equivalent to $S^{1} \times Q^{\wedge}\left(S^{1}\right)$. Then $f_{1}$ is the composite $Q^{\wedge}\left(S^{1}\right) \rightarrow Q\left(S^{1}\right) \rightarrow Q\left(D_{2}\right)$. It is immediate that $g_{1} f_{1}$ induces an isomorphism on $\pi_{2}$. Note that $f_{1}$ is not an $H$-map.

To define $f_{2}$, use the adjoint of James' stable retraction in [12]. Then $g_{2} f_{2}$ induces an isomorphism on $\pi_{3}$. Note that $f_{2}$ is not an $H$-map.

To define $f_{3}$, use the lift of the second Hopf invariant given in Lemma 3.1 of [6]. Again $g_{3} f_{3}$ induces an isomorphism on $\pi_{1}$.

To apply Corollary 6.2, we need to know that the $g_{i}$ exist. To obtain $g_{1}$, use the extension of $\Sigma R P^{\infty} \rightarrow Q\left(S^{1}\right)$ obtained by taking the adjoint of J. H. C. Whitehead's map $R P^{\infty} \rightarrow Q_{0}\left(S^{0}\right)$. To obtain $g_{2}$, use the extension of $\Sigma C P^{\infty} \rightarrow S U$ given in [13]. The construction of $g_{3}$ is the main result of [7]. 
We remark that one point of Corollary 6.2 is that the maps $g_{i}$ (and $f_{i}$ ) are essentially arbitrary. In particular, $g_{i}$ may not necessarily be an $H$-map. Kahn and Priddy [14, 15], J. F. Adams [1], and P. Loeffler and N. Ray [16] use a map $g_{1}$ which is an infinite loop map. The map $g_{2}$ can be traced to the work of I. M. James [12]. Later, G. Segal [18] proved that $B U$ is a retract of $Q\left(C P^{\infty}\right)$, and this follows by looping $g_{2}$. Since $g_{2}$ induces an isomorphism on rational homotopy, it follows that the other factor of $Q\left(\Sigma C P^{\infty}\right)$ has finite homotopy groups.

7. Theorem 2.8 and product decompositions. In this section, all spaces $X$ are localized at $p$ and are assumed to be of finite type over $Z_{(p)}$. Our main result is the following theorem.

THEOREM 7.1. If $\Sigma^{2} X$ is not homotopy equivalent to a sphere or a point, then $\Omega \Sigma^{2} X$ admits a nontrivial product decomposition up to homotopy.

This theorem was known to Cohen, Moore, and Neisendorfer and they gave a proof in an unpublished computation [9]. Some analogous decompositions and related applications are given in [5 and 10]. We note that Theorem 2.8 is an immediate corollary.

We first set up some notation and prove two lemmas. We require maps $\beta_{k}$ : $(\Sigma X)^{[k]} \rightarrow(\Sigma X)^{[k]}$, where $Y^{[k]}$ denotes the $k$-fold smash product of the space $Y$. Let $\sigma_{k}: Y^{[k]} \rightarrow Y^{[k]}$ denote the cyclic permutation $(1, k, k-1, \ldots, 2)$ and recall that $\left[(\Sigma X)^{[k]} ; Z\right]$ is a group for $k \geqslant 1$. Define $\beta_{k}$ inductively by setting $\beta_{2}=1-\sigma_{2}$ and $\beta_{k+1}=\left(1 \Lambda \beta_{k}\right)-\left(\beta_{k} \Lambda 1\right) \cdot \sigma_{k}$.

LEMMA 7.2 (DYNKIN-SPECHT-WEVER RELATION). $\beta_{k^{*}} \beta_{k^{*}}=k \beta_{k^{*}}$.

Proof. Let $M=\bar{H}_{*}(\Sigma X)^{[k]}$ and $[a, b]=a \otimes b-(-1)^{|a||b|} b \otimes a$ in $M \otimes M$. By definition, we have

$$
\beta_{k^{*}}\left(m_{1} \otimes \cdots \otimes m_{k}\right)=\left[m_{1}\left[m_{2}\left[\ldots\left[m_{k-1}, m_{k}\right] \ldots\right],\right],\right] .
$$

The lemma now follows from the computation in the proof of Theorem 8 of $[11, p$. 169].

Let $L_{k}(\Sigma X)$ and $M_{k}(\Sigma X)$ denote the mapping telescopes of $\beta_{k}$ and $k-\beta_{k}$ respectively. Define $\phi_{k}:(\Sigma X)^{[k]} \rightarrow L_{k}(\Sigma X) \vee M_{k}(\Sigma X)$ to be the composite $(\Sigma X)^{[k]} \rightarrow(\Sigma X)^{[k]} \vee(\Sigma X)^{[k]} \rightarrow L_{k}(\Sigma X) \vee M_{k}(\Sigma X)$. By Lemma 7.2, $\phi_{k^{*}}$ induces an isomorphism on $H_{*}(; Z[1 / k])$. Thus as in [4], we have proved the following lemma.

LEMMA 7.3. If $(k, p)=1$, then $\phi_{k}$ is a homotopy equivalence.

Proof of Theorem 7.1. We shall exhibit a loop map $\Omega(w): \Omega \Sigma L_{k}(\Sigma X) \rightarrow \Omega \Sigma^{2} X$ and a map $h: \Omega \Sigma^{2} X \rightarrow \Omega \Sigma L_{k}(\Sigma X)$ such that $h \cdot \Omega(w)$ induces an isomorphism on $H_{*}()$ if $(k, p)=1$. Thus $\Omega \Sigma L_{k}(\Sigma X)$ is a $p$-local retract of $\Omega \Sigma^{2} X$. We will show that $\Omega \Sigma^{2} X$ is homotopy equivalent to $\Omega \Sigma L_{k}(\Sigma X) \times Z$ for some $Z$. To prove Theorem 7.1, we must check that $L_{k}(\Sigma X)$ is more highly connected than $\Sigma X$ and that $\bar{H}_{*} L_{k}(\Sigma X)$ is nontrivial. But $L_{k}(\Sigma X)$ is a retract of $(\Sigma X)^{[k]}$ and so it is more 
highly connected than $\Sigma X$. Also, if $\Sigma X$ is not a sphere or a point, there exist at least two elements $u$ and $v$ in $\bar{H}_{*} \Sigma X$. Then $\operatorname{ad}^{k-1}(v)(u)$ is a nonzero element of $\bar{H}_{*} L_{k}\left(\sum X\right)$.

Next we show that $\Omega \Sigma^{2} X$ splits. Let $F$ be the homotopy fibre of $w$ and let $\pi$ : $\Omega \Sigma^{2} X \rightarrow F$ be the natural map obtained from the Barratt-Puppe sequence. There is a morphism of fibrations:

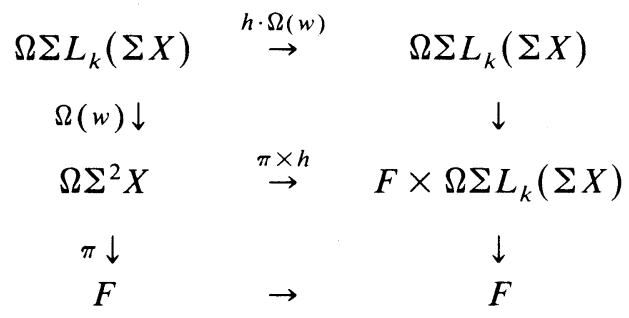

and so $\pi \times h$ induces a homology isomorphism.

Now we exhibit $\Omega(w)$ and $h$. Consider the Freudenthal suspension $E: \Sigma X \rightarrow$ $\Omega \Sigma^{2} X$ and the $k$-fold Samelson product $\operatorname{ad}^{k-1}(E)(E):(\Sigma X)^{[k]} \rightarrow \Omega \Sigma^{2} X$. Notice that this map induces the map $\beta_{k^{*}}$ in homology. Thus if $(k, p)=1$, then $\operatorname{ad}^{k-1}(E)(E)$ restricted to $L_{k}(\Sigma X)$ induces an isomorphism from $H_{*} L_{k}(\Sigma X)$ to the Lie tensors of length $k$. Let $\Omega(w): \Omega \Sigma L_{k}(\Sigma X) \rightarrow \Omega \Sigma^{2} X$ be the canonical multiplicative extension of the induced map $\operatorname{ad}^{k-1}(E)(E): L_{k}(\Sigma X) \rightarrow \Omega \Sigma^{2} X$. Finally, we construct $g: \Sigma \Omega \Sigma^{2} X \rightarrow \Sigma \Omega \Sigma L_{k}(\Sigma X)$ such that $g \cdot \Sigma \Omega(w)$ induces a homology isomorphism. It follows from Lemma 1.6 in [8] that there is a map $h$ such that $h \cdot \Omega(w)$ is an equivalence. To construct $g$, recall that $\Sigma \Omega \Sigma^{2} X$ is homotopy equivalent to $\Sigma\left(\bigvee_{j \geqslant 1}(\Sigma X)^{[j]}\right)$. Pinch the spaces $(\Sigma X)^{[j]}$ to a point if $j \neq 0 \bmod k$ to obtain a map $\Sigma \Omega \Sigma^{2} X \rightarrow \Sigma\left(\bigvee_{j \geqslant 1}(\Sigma X)^{[j k]}\right)$. Next, observe that by Lemma 7.3, $(\Sigma X)^{[j k]}$ is homotopy equivalent to $\left(L_{k}(\Sigma X) \vee M_{k}(\Sigma X)\right)^{[j]}$. Project $(\Sigma X)^{[j k]}$ to $\left(L_{k}(\Sigma X)\right)^{[j]}$. The composite of these maps gives $g: \Sigma \Omega \Sigma^{2} X \rightarrow \Sigma \Omega \Sigma L_{k}(\Sigma X)$ which by inspection has the property that $g \cdot \Sigma \Omega(w)$ induces a homology isomorphism.

\section{BIBLIOGRAPHY}

1. J. F. Adams, The Kahn-Priddy Theorem, Proc. Cambridge Philos. Soc. 73 (1973), 45-55.

2. H. E. A. Campbell, F. R. Cohen, F. P. Peterson and P. S. Selick, The space of maps of Moore spaces into spheres, Ann. of Math. Studies (to appear).

3. H. E. A. Campbell, F. P. Peterson and P. S. Selick, Self-maps of loop spaces. I, Trans. Amer. Math. Soc. 293 (1986), 1-39.

4. F. R. Cohen, Splitting certain suspensions via self-maps, Illinois J. Math. 20 (1976), 336-347.

5. __ Orders of certain compositions, Canad. Math. Soc. Conf. Proc., vol. 2, 1982, pp. 289-295.

6. The unstable decomposition of $\Omega^{2} \Sigma^{2} X$ and its applications, Math. Z. 182 (1983), 553-568.

7. Two-primary analogues of Selick's Theorem and the Kahn-Priddy Theorem for the 3-sphere, Topology 23 (1984), 401-421.

8. F. R. Cohen, J. C. Moore and J. A. Neisendorfer, Exponents in homotopy theory, Ann. of Math. Studies (to appear).

9. Homology and James-Hopf invariants (unpublished).

10. F. R. Cohen and J. A. Neisendorfer, A construction of p-local $H$-spaces, Lecture Notes in Math., vol. 1051, Springer-Verlag, Berlin and New York, 1984, pp. 351-359.

11. N. Jacobson, Lie algebras, Interscience, New York, 1962. 
12. I. M. James, Cross-sections of Stiefel manifolds, Proc. London Math. Soc. 8 (1958), 536-547.

13. , The topology of Stiefel manifolds, London Math. Soc. Lecture Note Series, no. 24, Cambridge Univ. Press, Cambridge, 1976.

14. D. S. Kahn and S. B. Priddy, Applications of the transfer to stable homotopy theory, Bull. Amer. Math. Soc. 78 (1972), 981-987.

15 . $103-111$

16. P. Loeffler and N. Ray, A geometric proof of a theorem of Kahn and Priddy, J. London Math. Soc. 24 (1981), 329-334.

17. J. C. Moore, Séminaire Henri Cartan, 1954/55, École Norm. Sup.

18. G. Segal, The stable homotopy of complex projective space, Quart. J. Math. Oxford Ser. (2) 24 (1973), $1-5$.

Department of Mathematics, Queens University, Kingston, Ontario, Canada

Department of Mathematics, University of Kentucky, Lexington, Kentucky 40506

Department of Mathematics, Massachusetts institute of Technology, Cambridge, MasSACHUSETTS 02139

Department of Mathematics, University of Toronto, Toronto, Ontario, Canada 